Journal of Non-Newtonian Fluid Mechanics, 1 (1976) 1-17

(C) Elsevier Scientific Publishing Company, Amsterdam - Printed in The Netherlands

\title{
TENSILE STRESS CONTRIBUTION OF FLOW-ORIENTED SLENDER PARTICLES IN NON-NEWTONIAN FLUIDS
}

\author{
J.D. GODDARD
}

University of Michigan, Department of Chemical Engineering, Ann Arbor, Michigan 48104 (USA)

(Received March 4, 1975)

Summary

A formula is derived for the intrinsic stress contribution from closelyspaced, rod-like rigid particles suspended in a non-Newtonian liquid subject to a simple extensional flow.

The present results indicate that, compared to the Newtonian-fluid result given previously by Batchelor [1], a much smaller particle-stress effect may occur in non-Newtonian fluid, owing to shear thinning and possible tensile stiffening in the fluid itself. While unsubstantiated by any critical experimental test, this prediction appears to agree qualitatively with some recent experimental observations of Charrier and Rieger [29] on glass fibers in polymer melts.

Further work is suggested for the relevant slender-body theory, particularly the far-field body influence in non-linear fluids.

\section{Introduction}

Suspensions of solid particles in liquids represent an important class of non-Newtonian fluids to which there has been a good deal of effort devoted, in both theoretical and experimental rheology. An especially interesting subclass of such suspensions are those containing particles of a fibrous or rod-like form. Such fluids are found in several natural and technological settings, with particles or fibers ranging in size from macroscopic, down to microscopic dimensions.

Based on several theoretical studies and some recent experiments $[1,2,3,4]$ it appears that such suspensions can be expected to exhibit rather significant non-Newtonian effects and, moreover, large stress contributions from the particles, especially when the particles are oriented by flow or other processes.

These, and related considerations have provided impetus to recent developments in the slender-body theory of small particles in viscous Newtonian fluids $[5,6,7,8]$. 
There exist, however, some practically interesting fluid suspensions in which the suspending fluid itself is distinctly non-Newtonian in character, even without particles. Apart from the recent work of Leal [9], on the behavior of isolated particles in shear flow, and that of Lockett [10] on concentrated suspensions in simple shear, both of which adopt a second-order fluid model for describing the micro-mechanics, there has been little done in the way of theory for slender particles in non-Newtonian fluids.

This provides much of the motivation for the present work, in which we consider one of the most elementary flows of a suspension, consisting of a uniaxial extension, or "axisymmetric pure straining", of the type that may be expected to occur in some technological processes, such as fiber spinning, capillaryentrance flow and, possibly, injection moulding of polymers.

Following Batchelor's Newtonian analysis [11], which shows dramatic stress effects in such flows and which has been the subject of some partial experimental applications and confirmation $[2,3,4]$, we undertake here to exploit some of the simplifications inherent to the description of highly oriented suspensions of slender particles, in order to analyze non-Newtonian suspending media as well. To the extent that these simplifications are geometric in origin, a characteristic of slender-body theory in general, one expects of them a degree of universal validity irrespective of the materials involved.

For this reason, we have attempted to provide a reasonably general discussion of certain salient aspects of the relevant theory for non-Newtonian fluids without, at the outset, making very definite or restrictive constitutive assumptions for the fluid. In this way, we are able to point out some of the major distinctions from the previous Newtonian analyses, and to isolate particular aspects of the theory which need further development.

Also, we are able to provide an extension of the Newtonian result of Batchelor [11] for the stress in closely-spaced particle suspensions, which requires reasonably simple rheological information on the shear and extensional behavior of the non-Newtonian fluid to make it definite. Based on certain assumed representations of such behavior, it appears that one may expect to obtain fairly significant differences between the Newtonian and non-Newtonian cases.

Thus our aim is to determine the contribution to the bulk or macroscopic stress due to the presence of highly-elongated rigid particles in an (incompressible) non-Newtonian liquid subjected to a uniaxial or "simple" extension. In the absence of particles, the undisturbed fluid-velocity field is assumed to have the form:

$V=E \cdot x$

where, relative to an appropriate set of cartesian axes $x, y, z$ fixed in the material, the velocity-gradient tensor is identical with the rate of deformation tensor $E$, which in turn may be taken to have the purely diagonal matrix of components

$$
[E]=\left[\begin{array}{lll}
-\dot{\epsilon} / 2 & 0 & 0 \\
0 & -\dot{\epsilon} / 2 & 0 \\
0 & 0 & \dot{\epsilon}
\end{array}\right]
$$


That is, in terms of the components of $V$

$V_{x}=\dot{\epsilon} x / 2 \quad V_{y}=-\dot{\epsilon} y / 2 \quad V_{z}=\dot{\epsilon} z$

where $z$ denotes the axis of extension and $\dot{\epsilon}(>0)$, independent of position, denotes the rate of extension or elongation. Although we shall focus attention here on the case of time-independent $\dot{\epsilon}$, the analysis would also be applicable to quasi-steady extensions, where $\dot{\epsilon}$ is a sufficiently slowly varying function of time (relative to an appropriate relaxation time for the fluid).

In any case, since we are mainly concerned with local flow in the vicinity of "small" suspended particles (small compared to typical macroscopic length scales but large compared to the dimensions of molecules in the suspending fluid), we shall adopt the assumption, common to most suspension theories, of "creeping" or inertialess motion. This assumption must of course rest ultimately on the criterion of a sufficiently small value of an appropriate dimensionless number, the analogue of the particle Reynolds-number criterion for a Newtonian fluid.

The present analysis is also based on the assumption of elongated particles having an extreme form, with one characteristic (length) dimension $2 l$, say, much greater than the typical breadth dimension $2 a$, say. That is, in terms of an axial ratio $\alpha$

$\alpha \equiv l / a \gg 1$

This leads to the type of slender-body problem already treated by Batchelor $[1,11]$ for the Newtonian fluid. As he points out in that context, one may expect that an elongated rigid body having sufficient fore-aft symmetry will tend to align itself with its major dimension $2 l$ parallel to the axis of extension $z$, when placed in a flow field having the (undisturbed) form of eqn. (3), as illustrated in Fig. 1. (We assume here that orientation effects due to Brownian motion or to external torques and forces are negligible.) Although we expect the same to be true for a fairly wide class of non-Newtonian or viscoelastic fluids, and shall assume it to be the case here, the necessary restrictions on particle shape or symmetry may be more severe than in the Newtonian case, particularly because of non-linear rheological effects, of a kind to be discussed below. Precisely because of such effects, and for the sake of being definite, we shall eventually restrict our analysis to axially-symmetric particles with fore-aft symmetry, in which case $l$ shall denote the semi-major axis and $a$ some characteristic particle radius, say in its midplane $z=0$.

Within this framework, we wish to consider the asymptotic form for $\alpha \rightarrow \infty$ of the velocity field and stress the neighborhood of a given particle. In view of the previous experience with the corresponding problems for the Newtonian fluid $[5,6,11,12]$ or Hookean solid [13], one expects that it should be possible to formulate the present problem as a singular perturbation scheme, involving both "inner" and "outer" expansions for velocity and stress fields. The evident distinction here is that, without the benefit of linearity in the basic field equations, i.e., the Stokes equations for a Newtonian fluid, one cannot hope to achieve a complete theory for general non-linear viscoelastic fluids. Furthermore, 


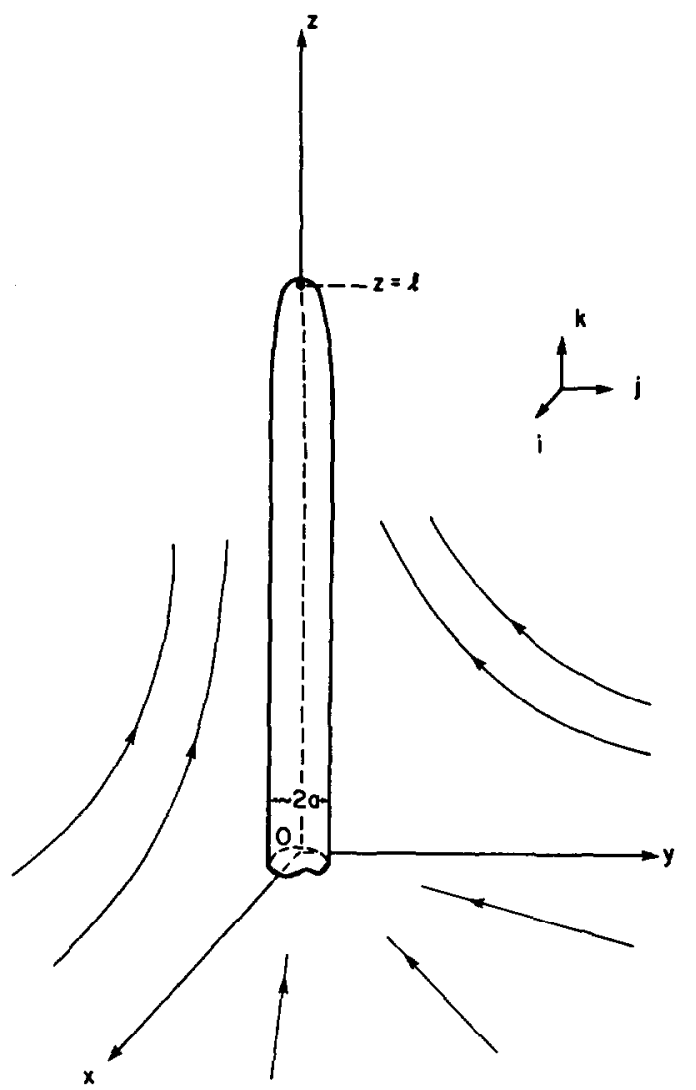

Fig. 1. Half-space $z>0$, showing upper part of an oriented slender body and streamlines of the undistributed flow $V$.

in this type of problem, the possibility of particle interactions, even in nominally dilute systems, poses an immediate difficulty in the description of the "far field" and, hence, in the definition of a proper outer expansion. In the context of slender particles oriented by an uniaxial extension, Batchelor [1] has given a fairly comprehensive discussion of the Newtonian-fluid problem, in which he considers in detail the respective cases of isolated, individual particles where interactions between particles are negligible, and closely-spaced parallel particles, where such interactions are dominant in the far field.

For these reasons, and with the modest aim of obtaining only the leading or "zeroth-order" approximation for $\alpha \rightarrow \infty$, we adopt here a somewhat different approach from that of the above authors. In particular, and from the outset, we shall focus attention mainly on the leading terms in the inner flow field, anticipating that the appropriate outer boundary conditions are to be provided by the appropriate matching with an outer flow of the form of eqn. (1). In Section 3, then, we shall consider the approximate "cell-model" boundary conditions appropriate to "closely-spaced" particle suspensions, of the type considered by Batchelor [1].

As for fluid rheology, we shall merely assume, for the present purposes, 
that the fluid falls into the category of "simple fluids with fading memory" $[14,15]$, which appears to be the case for most of the (integral or differential) models that have been proposed to represent polymer fluids. In the present context, this assumption would at least allow for various well-known rheological representations, such as the Rivlin-Ericksen expansions.

\section{The asymptotic form of the inner flow near a slender particle}

We consider first the fluid velocity field in the vicinity $\left(|z|<l\right.$ and $x^{2}+$ $y^{2} \ll l^{2}$ ) of a particle in the assumed equilibrium orientation, as shown schematically in Fig. 1. Provided the cross-sectional form of the particle varies sufficiently slowly with axial ( $z$ ) position ( $c f$. Clarke [8] and Batchelor [11]), it seems reasonable at the outset to adopt dimensional scaling typical of the Newtonian-fluid theory for the velocities and velocity gradients.

Thus, we now distinguish dimensional quantities by asterisks and let

$x^{*}=a x \quad y^{*}=a y \quad z^{*}=l z$

$v_{x}^{*}=a \dot{\epsilon} v_{x} \quad v_{y}^{*}=a \dot{\epsilon} v_{y} \quad v_{z}^{*}=l \dot{\epsilon} v_{z}$

We are concerned then with the limiting forms of the dimensionless velocities $v_{x}, v_{y}, v_{z}$, and their $x, y, z$ derivatives for $1 / \alpha \rightarrow 0$ in an inner region near the body, where $x, y, z$ are all $\mathrm{O}(1)$. Since the gradient operator is given by:

$$
\begin{aligned}
\nabla^{*} & =i \frac{\partial}{\partial x^{*}}+j \frac{\partial}{\partial y^{*}}+k \frac{\partial}{\partial z^{*}} \\
& =\frac{1}{a}\left\{\nabla^{(0)}+\frac{1}{\alpha} \nabla^{(1)}\right\}
\end{aligned}
$$

where:

$$
\begin{aligned}
& \nabla^{(0)}=i \frac{\partial}{\partial x}+j \frac{\partial}{\partial y} \\
& \nabla^{(1)}=k \frac{\partial}{\partial z}
\end{aligned}
$$

one has formally for the velocity gradient

$$
\begin{aligned}
\nabla^{*} v^{*} & =\alpha \dot{\epsilon}\left[\nabla^{(0)}\left(k v_{z}\right)+O(v / \alpha)\right] \\
& =\alpha \dot{\epsilon}\left[i k \frac{\partial v_{z}}{\partial x}+j k \frac{\partial v_{z}}{\partial y}+O(v / \alpha)\right]
\end{aligned}
$$

Here, we denote by $\mathrm{O}(v / \alpha)$ terms which are $\mathrm{O}(1 / \alpha)$ relative to the leading terms in eqn. (9). The leading terms themselves are seen to represent a rectilinear flow composed of simple shears, and, hence the inner flow field is "quasi-viscometric" in character. 
As in other such slender-body approximations, the higher-order terms in eqn. (9) involve derivatives $\partial / \partial z$ and related terms, which can become large in regions where the body cross-section changes rapidly in the axial direction and, also, near the ends $z= \pm 1$ of the body. If, as indicated above, we restrict our attention to sufficiently smooth bodies, then the end-effects are expected to make negligible contributions, beside those of the leading terms, to the quantities of primary interest here, such as the fluid drag and its integral moments on the upper $(z>0)$ or lower $(z<0)$ portions of the body. *

While the above reasoning leads basically to the same type of zeroth-order inner approximation for the velocity gradient as that obtained previously for the special case of a Newtonian fluid, there are some distinctions to be made, relative to the magnitudes of terms involved and to the compatibility with the equations of motion.

We recall that, for the Newtonian case, one finds the terms indicated by $\mathrm{O}(v / \alpha)$ preceded by larger terms involving a parameter of the form $1 / \log \alpha$, which results essentially from the nature of the solution to the Stokes equations [ 5,11$]$. That is to say, retention of the leading terms from eqn. (9) in the relevant equations of motion leads to a zeroth approximation to $v_{z}(x, y, z)$, say $v_{z}^{(0)}(x, y, z)$, which involves a relative error $\mathrm{O}(1 / \log \alpha)$, a quantity much larger than $\mathrm{O}(1 / \alpha)$, for $1 / \alpha \rightarrow 0$.

In the case of a non-Newtonian fluid we may expect to encounter the same type of phenomenon, although the order of magnitude of the leading terms and the form of the appropriate error term or perturbation parameter will depend on the fluid rheology, and, in particular, on its simple-shear behavior, as will be reconsidered briefly below. However, for the present purposes we shall not bother to make any explicit notational distinction between the exact velocity component $v_{z}(x, y, z)$ and its zeroth approximation for $1 / \alpha \rightarrow 0$.

The second consideration which arises in the case of non-Newtonian fluids relates to the rheological compatibility of the postulated flow field with the equations of motion. Since this depends crucially on the type of fluid being considered, some further delineation of rheological effects is called for. To this end, we note that, with the above-mentioned restriction on body smoothness, we may regard the leading term in eqn. (9) as a quasi-steady viscometric flow provided the fluid has a sufficiently rapid fading memory, that is, a sufficiently small relaxation time, at the shear-rate levels of interest. This follows from the fact that the (substantial or material) times rates of change, represented by quantities like:

$v_{z}^{*} \frac{\partial}{\partial z^{*}}\left(\frac{\partial v_{z}^{*}}{\partial x^{*}}\right) \equiv \alpha \dot{\epsilon}^{2} v_{z} \frac{\partial^{2} v_{z}}{\partial z \partial x}$

* The influence of "end-effects", for certain bluff bodies, on the higher-order approximations in slenderness have been recently put into perspective by the works of Tillet [12] and others; $c f$. also Russel and Acrivos [13]. In addition, Weinberger [16] has considered the effects of certain extreme non-uniformities of body shape in the "zeroth order." In the present context, an accounting for such higher-order terms and extremities of shape must, unfortunately, await further developments in the associated non-linear field theory. 
are $O(1 / \alpha)$ compared to quantities like

$$
\left(\frac{\partial v_{z}^{*}}{\partial x^{*}}\right)^{2}=\alpha^{2} \dot{\epsilon}^{2}\left(\frac{\partial v_{z}}{\partial x}\right)^{2}
$$

As a consequence, any of the higher (invariant) time derivatives of strain rate, for example, the higher-order Rivlin-Ericksen tensors [15,17] are expressible, with a relative accuracy $O(1 / \alpha)$, in terms of quantities of the latter kind above, exactly as in a steady rectilinear flow. Hence, it is easy to show that a Rivlin-Ericksen fluid of any order would exhibit the stress pattern associated with a steady viscometric flow, and we expect the same to be true of sufficiently smooth, "simple" fluids in general. (Also, we observe that there is some experimental evidence to suggest that polymer fluids exhibit more rapidly fading memory, or shorter relaxation times, against small perturbations, the larger the basic shear rate involved $[3,18,19])$.

Then, to be consistent with the quasi-steady viscometric flow represented by the leading terms of eqn. (9), a simple fluid will exhibit the corresponding stress pattern [20] given by a stress tensor $T^{(0)}$, say, as:

$$
\begin{aligned}
T^{(0)}= & \eta(\dot{\gamma})\left[(i k+k i) \dot{\gamma}_{x}+(j k+k j) \dot{\gamma}_{y}\right]+ \\
& +\frac{N_{2}}{\dot{\gamma}^{2}}\left[\dot{\gamma}_{x}^{2} i i+\dot{\gamma}_{x} \dot{\gamma}_{y}(i j+j i)+\dot{\gamma}_{y}^{2} j j\right]+k k\left(N_{1}+N_{2}\right)-p 1
\end{aligned}
$$

where:

$\dot{\gamma}_{x}=\alpha \dot{\epsilon} \frac{\partial v_{z}}{\partial x} \quad \dot{\gamma}_{y}=\alpha \dot{\epsilon} \frac{\partial v_{z}}{\partial y} \quad \dot{\gamma}^{2}=\dot{\gamma}_{x}^{2}+\dot{\gamma}_{y}^{2}$

$p$ and 1 denote respectively an isotropic pressure and the unit tensor, and where $\eta(\dot{\gamma}), N_{1}(\dot{\gamma})$ and $N_{2}(\dot{\gamma})$ are respectively the viscometric material functions: the apparent viscosity, and the primary and secondary normal stress, all even func. tions of the shear rate $\dot{\gamma}$. Furthermore, and consistent with the form of the gradient operator in eqn. (7), the relevant equation of inertialess motion is taken to be

$\nabla^{(0)} \cdot T^{(0)}=0$

In writing down eqn: (12), which involves a rheologically indeterminate pressure term, we have anticipated an eventual matching of zeroth-order terms with an essentially constant pressure in the far field.

Then, with the form of eqn. (10) for $T^{(0)}$, this reduces to a set of well-known equations for rectilinear flow governing the shear-stress and normal-stress terms, respectively. The first of these has the form

$$
\frac{\partial}{\partial x}\left(\eta \dot{\gamma}_{x}\right)+\frac{\partial}{\partial y}\left(\eta \dot{\gamma}_{y}\right) \equiv \frac{\partial}{\partial x}\left(\eta \frac{\partial v_{z}}{\partial x}\right)+\frac{\partial}{\partial y}\left(\eta \frac{\partial v_{z}}{\partial y}\right)=0
$$


whereas the normal-stress equations reduce, upon elimination of the pressure gradient $\nabla^{\left({ }^{2}\right)} \dot{p}$, to the form

$\frac{\partial^{2}}{\partial x \partial y}\left[\frac{N_{2}\left(\dot{\gamma}_{x}^{2}-\dot{\gamma}_{y}^{2}\right)}{\dot{\gamma}^{2}}\right]=\left[\frac{\partial^{2}}{\partial x^{2}}-\frac{\partial^{2}}{\partial y^{2}}\right]\left[\frac{\dot{\gamma}_{x} \dot{\gamma}_{y} N_{2}}{\dot{\gamma}^{2}}\right]$

Equations (13) and (14) are special cases of those investigated by Ericksen [21] and Stone [22] * corresponding to a zero axial pressure gradient. It follows from their work that these equations are not compatible unless:

$N_{2} / \dot{\gamma}^{2} \eta(\dot{\gamma})=$ constant

or, otherwise, unless the flow field is either planar or, more relevant here, axisymmetric in form:

$v_{z}(x, y, z)=u(r, z)$

with

$r^{2}=x^{2}+y^{2}$

Rigorously speaking, then, unless we limit ourselves to axisymmetric particles the inner flow field of eqn. (9) cannot be compatible with the equations of motion, without the further restriction of eqn. (15) on fluid rheology, a restriction which limits one in principle to the "second-order" fluid regime.

From the point of view of obtaining a proper slender-body approximation in the present context, the above considerations imply that we should have to reformulate the order of magnitude analysis of eqn. (6) to allow for the possibility of lateral velocities $v_{x}, v_{y}$ which are not necessarily negligible in the zerothorder approximation, for $1 / \alpha \rightarrow 0$. Thus, in this approximation, the inner flow could no longer be considered viscometric, as suggested by eqn. (9), but, rather, would involve circulation or general transverse flow in planes $z=$ constant, of the type that has been predicted theoretically for the flow in non-circular tubes and similar geometries, e.g., by Green and Rivlin [24] and Wineman and Pipkin [25].

Based on the form of eqn. (15), and on a number of such theoretical studies which proceed via retarded-motion (Rivlin-Erickson) expansions about the rest state of the fluid, one expects such normal-stress effects to become operative in non-axisymmetric flows only at the fourth-order in shear rate $\dot{\gamma}$, that is, in the "fourth-order-fluid" approximation. As for the real importance of such effects in the non-linear viscometric regime for polymer fluids, we can state only that the secondary normal stress $N_{2}$ appears (by comparison to the shear stress and the primary normal stress) to be a relatively small quantity, approximately in accordance with the "Weissenberg hypothesis", $N_{2} \equiv 0$.

\footnotetext{
* cf. Fredrickson [23], p. 84, after correction of a misprint in his eqn. (4.77), and Truesdell [15].
} 
It is perhaps not unrealistic to expect, then, that for a rather broad class of non-Newtonian fluids the departures from our original approximation of eqn. (9), arising from the secondary normal stress, would represent small perturbation on a viscometric flow, essentially because of rheological behavior alone. *

At any rate, for the sake of simplicity, we shall henceforth limit the present discussion to axisymmetric particles where one can more justifiably ignore such effects of secondary normal stress on the zeroth-order inner flow.

For the axisymmetric problem, the axial velocity is given by eqn. (16), which satisfies eqn. (14) identically, and eqn. (13) takes on the simpler form:

$\frac{1}{r} \frac{\partial}{\partial r}\left(\eta r \frac{\partial u}{\partial r}\right)=0 \quad$ or $\quad \frac{1}{r} \frac{\partial}{\partial r}(r r)=0$

where, now, the quantities:

$\dot{\gamma}=\alpha \dot{\epsilon} \frac{\partial u}{\partial r}$ and $\tau=\eta(\dot{\gamma}) \dot{\gamma}$

with $\tau$ representing the relevant $(r z)$ shear stress, are both dimensional.

To define the zeroth-order terms in the inner flow completely, some boundary conditions are now in order. To this end, we require the velocity $u(z, r)$ to satisfy the usual adherence condition, $u=0$, on the particle surface, $r=R(z)$, say. In addition, and as mentioned above, we must have recourse to some type of outer boundary condition, in principle to be determined by the appropriate matching with a far-field or outer expansions for the velocity and stress. We recall that, for the isolated particle in an infinite body of Newtonian fluid, the far-field velocity distribution appears as a perturbation on the basic flow given by eqn. (1) and is determined by a "Stokeslet" distribution on the particle axis, which is in turn related directly to the particle surface stress associated with the inner flow field $[5,6,11]$. However, in the non-Newtonian case one does not have recourse to the underlying linear-superposition techniques nor to the resultant integral equations, so that it is not immediately evident how direct progress can be made on the isolated-particle problem in any general way. By contrast, in the other situation of closely-spaced particles, one can with certain reservations apply the approximate "cell" model proposed by Batchelor [1], which we consider presently.

First, however, we note that eqn. (17) can be integrated directly to give a familiar elementary form for the shear stress $\tau$ :

$\tau(r, z)=\tau_{R}(z) \frac{R(z)}{r}$

* However, the possible influence of $\mathrm{N}_{2}$ on particle orientation in similar contexts should should not be dismissed lightly. Thus, Leal [9] has recently offered a theoretical explanation based on $\mathrm{N}_{2}$ of the experimentally observed "orbit dirft $t^{\text {" }}$ of slender particles suspended in shear flows; although, it should be noted that such flows involve a basic farfield cross flow to the particle axis which, in contrast to the present situation, is intrinsic to the zeroth-order inner flow near the body. 
where $\tau_{R}(z)$ is the (unknown) stress at the particle surface, $r=R(z)$. Assuming, then, that the rheological relation represented by the second equation of eqn. (18) can be inverted to give the shear rate as a function $\dot{\gamma}\{\tau\}$ of the shear stress, one sees that the first equation of eqn. (18) can then be explicitly integrated, with a change of variables $r \rightarrow \tau$ given by eqn. (19) and the adherence condition at $r=R(z)$, to yield

$u(r, z)=\int_{R(z)}^{r} \dot{\gamma}\{\tau(r, z)\} \mathrm{d} r=\tau_{R} R \int_{R \tau_{R} / r}^{\tau_{R}} \dot{\gamma}\{\tau\} \mathrm{d} \tau$

This, of course, provides a definite relation between the axial velocity distribution and the particle-surface stress $\tau_{R}(z)$, once the surface shape $R(z)$ and the shear rheology of the fluid are known.

At the same time, it becomes evident that a further ("outer") boundary condition, e.g. on $u(r, z)$, is necessary to determine $\tau_{R}(z)$ and, hence, the effective axial tensile stress, say $\sigma(z)$, acting in the interior of the particle. Before proceeding to a definite outer boundary condition, we note that the stress $\sigma$ is determined by an elementary force balance on the particle as:

$\mathrm{d} F(z)=\mathrm{d}\left[\pi a^{2} R^{2}(z) \sigma(z)\right]=2 \pi a l R(z) \tau_{R}(z) \mathrm{d} z$

which represents an axial force distribution $\mathrm{d} F(z)$ and which, in the Newtonian case, represents the relevant "Stokeslet" distribution (cf. Batchelor [11]). * Consistent with our neglect of end-effects, we assume the particle tensile stress to vanish at its (hypothetical) ends, $z= \pm 1$, to yield, by eqn. (21):

$\sigma(z)=\frac{2 \alpha}{R^{2}(z)} \int_{z}^{1} R\left(z^{\prime}\right) \tau_{R}\left(z^{\prime}\right) \mathrm{d} z^{\prime}$

where $R(z)$ is an even function of $z$ and $\tau_{R}(z)$ is presumably odd, owing to the assumed symmetry of the system.

3. The cell model for the intrinsic particle stress in a closely-spaced particle suspension

For the Newtonian fluid, Batchelor [1] has given a rather detailed discussion of a suspension, subjected to a bulk or macroscopic strain rate of the form (1), wherein the particles are (statistically) arranged such that the mean spacing between particles, $b$ say, is related to the particle dimensions by:

$a \ll b \ll l$

\footnotetext{
* We assume that we may neglect those radial normal-stress contributions to axial stress arising from any inclination of the particle surface, since these involve terms of the form $\mathrm{O}(1 / \alpha) \mathrm{d} R / \mathrm{d} z$.
} 
that is:

$1 \ll \beta \ll \alpha$

where:

$\beta=\frac{l}{b}=\alpha \sqrt{\frac{\phi}{\pi}}$

with $\phi$ denoting the volume fraction of particles in the suspension.

Based on certain statistical assumptions and the linearity of the Newtonian equation of motion (the Laplace equation which results from taking $\eta$ to be constant in eqn. (13)), Batchelor [1] has put forth arguments for the approximate validity of the following "cell-model" boundary condition for eqn. (13) or eqn. (17):

$v_{z}^{*}=V_{z}=\dot{\epsilon} z^{*} \quad$ at $\quad r^{*}=b$

or:

$u(r, z)=V_{z} / \dot{\epsilon} l=z \quad$ at $\quad r=\frac{b}{a}$

for a representative particle situated, with midpoint at $z=0$ (as in Fig. 1), relative to a bulk flow given by eqn. (3). That is, the velocity disturbances due to individual particles are assumed to cancel one another, on the average, at a radial distance (from a given particle) characteristic of the mean particle spacing.

While the same assumption will be adopted here, the supporting arguments cannot be made so plausible as for the Newtonian case owing basically, once again, to the non-linearities in fluid behavior.

For example, we cannot generally apply Batchelor's elementary argument [1] for an isolated parallel pair of nearly grazing particles, in which he shows that occasional close encounters make no significant alterations in the result for bulk stress, at least, whenever particle end-effects are negligible.

In the second- or third-order-fluid approximation it appears plausible that such arguments might carry over to the non-Newtonian case, but, in general, the presence of normal-stress effects combined with the non-axisymmetric character of the flow near adjacent parallel particles could lead to significant departures from rectilinear flow, exactly as discussed above for the case of the single nonaxisymmetric particle, If so, then in addition to possible particle end-effects, a transverse flow throughout the region of proximity between nearly grazing particles could possibly result in significant effects on particle orientation, both on their separation distance and their tendency to remain parallel.

In addition to these difficulties, one must further acknowledge that, compared to the Newtonian case, eqn. (25) does not enjoy the same status as an approximate outer boundary condition, since it does not represent to the same degree an explicit matching of the inner and outer stress fields.

Bearing in mind these reservations as a motivation for further work on the 
theoretical problems involved, we shall tentatively adopt here the boundary conditions of eqn. (25). In this way, we can at least point up some of the significant differences to be expected between non-Newtonian and Newtonian suspending fluids, whenever conditions are such that the cell model can be considered approximately valid.

The intrinsic particle stress

As has been shown in previous works $[7,26]$ the proper expression for macroscopic stress in the present context has the form:

$\langle\sigma\rangle=\langle\sigma\rangle_{f}(1-\phi)+\langle\sigma\rangle_{p} \phi$

where $\phi$ denotes, as before, the volume fraction of particles, and \langle\rangle$,\langle\rangle_{f},\langle\rangle_{p}$ denote volume averages, respectively, over the whole volume of suspension, the region occupied by fluid alone, and that occupied by the particles. Also, $\sigma$ refers to the principal stress difference $\left(T_{z z}-T_{r r}\right)$ for the axisymmetric extension under consideration.

In the treatments referred to above, the suspending fluid is assumed Newtonian and, consequently, it is possible effectively to eliminate the term $\langle\sigma\rangle_{f}$, through the relation:

$\langle\sigma\rangle_{f}(1-\phi)=\sigma_{0}$

where $\sigma_{0}$ denotes the stress in the pure suspending fluid arising from the given macroscopic strain, when $\phi=0$. This follows simply from the fact that the effective strain rate $E$ is given in general by:

$E=\langle E\rangle_{f}(1-\phi)+\langle E\rangle_{p} \phi$

from which the second term vanishes for rigid particles (cf. Goddard and Miller, [26]).

Hence, if one defines an intrinsic (particle) stress $[\sigma]$ as the fractional increase in $\langle\sigma\rangle$ due to the presence of particles, per unit volume-fraction particles, we have

$[\sigma] \equiv \frac{\langle\sigma\rangle-\sigma_{0}}{\phi \sigma_{0}}$

Apart from a constant factor this corresponds to the usual definition of "intrinsic viscosity" (which represents here the tensile or extensional viscosity) and, for a Newtonian suspending fluid, is quite simply given by eqns. (26) and (27) as

$[\sigma]=\langle\sigma\rangle_{p} / \sigma_{0}$

This simple result, based on eqn. (27), is a direct consequence of linearly viscous behavior and, unfortunately, does not carry over to the non-Newtonian fluid. 
However, based on the Newtonian analysis of Batchelor [1] for closelyspaced particles, one is led to propose tentatively that in many situations of practical interest the particle stress term $\langle\sigma\rangle_{p} \phi$ in eqn. (26) will be predominant.

Thus, we are led to adopt eqn. (30) as an estimate for the intrinsic particle stress in the non-Newtonian case as well, provided that it is a large quantity; that is, provided

$\langle\sigma\rangle_{p} \gg \sigma_{0}$ and, hence, $[\sigma] \gg 1$

For closely-spaced slender particles the validity of this approximation will be strongly influenced by the shear and extensional theology of the non-Newtonian fluid, as will now be considered in more detail.

A specific rheological model and some implications

By means of eqn. (22), the volume average particle stress:

$\langle\sigma\rangle_{p}=\int_{0}^{1} \sigma(z) R^{2}(z) \mathrm{d} z / \int_{0}^{1} R^{2}(z) \mathrm{d} z$

can be expressed as a double integral which, in turn, can readily be reduced to the single integral:

$\langle\sigma\rangle_{p}=2 \alpha \int_{0}^{1} R(z) \tau_{R}(z) z \mathrm{~d} z / \int_{0}^{1} R^{2}(z) \mathrm{d} z$

a result which might also have been deduced from a formula of Batchelor [11].

By applying, then, the cell-model boundary condition of eqn. (25) in eqn. (2), one obtains the further relation

$z=\frac{R(z) \tau_{R}(z)}{\alpha \dot{E}} \int_{(R \sigma / b) \tau_{h}}^{\tau_{R}} \dot{\gamma}\{\tau) \frac{c^{\frac{\pi}{\sigma}}}{\tau^{2}}$

which, as anticipated in the paragraph following eqn. (20), provides an implicit equation for the shear stress distribution $\tau_{R}(z)$ in eqn. (33), depending on the shear theology, through $\dot{\gamma}\{\tau\}$; the particle shape through $R(z)$; and the volume fraction $\phi$, through $a / b=\beta / \alpha$ and eqn. (24).

Because of their implicit nature, the above relations are not especially illuminating, and to obtain a more definite result with potential applicability to polymer fluids we consider as a specific rheological form, the familiar "spowerlaw model" for shear behavior:

$\tau\{\dot{\gamma}\} \propto \dot{\gamma}^{n+1} \quad$ or $\quad \dot{\gamma}\{\tau\} \propto \tau^{\frac{1}{n+1}}$ 
Here, we take $\dot{\gamma}$ and $\tau$ to be essentially positive quantities and $-1<n<0$, corresponding to the situation of most practical interest, the so called shear-thinning or "pseudo-plastic" behavior.

Substitution of eqn. (35) into eqn. (34) leads then to an expression for shear rate at the particle-surface:

$\dot{\gamma}_{R}(z)=\frac{\alpha z \dot{\epsilon}}{R}\left[\frac{-n}{(n+1)\left\{1-(R a / b)^{\frac{-n}{n+1}}\right\}}\right]$

displaying an approximate linear increase with $z$, which becomes exact for particles of constant circular cross-section, $R \equiv 1$. At any rate, one obtains from eqn. (36), for the ratio in eqn. (31):

$\left\langle\sigma_{p}\right\rangle / \sigma_{0}=g_{n}(a / b) h(\dot{\epsilon})$

where:

$h(\dot{\epsilon})=\frac{3 \tau\{\dot{\epsilon}\}}{\sigma_{0}\{\dot{\epsilon}\}}$

and

$g_{n}=\frac{2 \alpha}{3} \int_{0}^{1}\left[\frac{-n \alpha}{(n+1)\left\{1-(R a / b)^{\frac{-n}{n+1}}\right\}}\right]^{n+1}\left(\frac{z}{R}\right)^{n+2} R^{2} \mathrm{~d} z / \int_{0}^{1} R^{2} \mathrm{~d} z$

Here, $g_{n}$ is a factor dependent on the particle geometry and concentration as well as on the fluid rheology (through $n$ ).

On the other hand, the second factor $h(\dot{\epsilon})$, which depends only on fluid rheology, merits further consideration. Thus, we note that the quantity $\sigma_{0}\{\dot{\epsilon}\}$ in eqn. (37) is to be interpreted as the tensile stress, as a function of the extension rate $\dot{\epsilon}$, for the pure suspending fluid, whereas $\tau\{\dot{\epsilon}\}$ denotes the shear-stress function, which has the form of eqn. (35) and is to be evaluated here at shear rate $\dot{\gamma} \equiv \dot{\epsilon}$. Therefore, the quantity $h(\dot{\epsilon})$ in eqn. (37) can merely be regarded as thrice the ratio of the apparent shear viscosity to the tensile viscosity, both evaluated at $\dot{\epsilon}$, as such, is identically equal to unity for the Newtonian case. (cf. Weinberger and Goddard [3], Batchelor [1].)

For non-Newtonian fluids, however, the presence of this peculiar stress ratio $h(\dot{\epsilon})$ has interesting implications, insofar as it governs the particle-stress contribution in a very direct manner. In this regard, there are some experimental indications that certain polymer fluids, such as the solutions studied by Metzner and Metzner [27] and by Weinberger and Goddard [3], may exhibit vastly greater stress levels in extension than in shear, implying that the factor $h(\dot{\epsilon})$ in question can be an exceedingly small quantity. If so, this would imply, by eqn. (37), a drastically reduced intrinsic stress for fibrous particles suspended in such fluids, as compared to similar suspensions in Newtonian fluids.

Of some practical interest is the case of molten polymers, to which small 
solid fibers are sometimes added to provide reinforcement of an eventual, solid product. For these fluids there are various experimental studies * which indicate that the stress ratio $h$ may either be large or of order unity [28] for polymer "melts". The case of small $h$ would appear to have further practical significance, since, to the extent that solid polymers can be considered approximately incompressible and Hookean at small strains, the Newtonian form of eqn. (37) for $n \rightarrow 0$ (cf. Batchelor, [1]):

$\langle\sigma\rangle_{p} / \sigma_{0}=g_{0}=\frac{2 \alpha^{2}}{3} \int_{0}^{1} \frac{z^{2} \mathrm{~d} z}{\ln (b / a R)} / \int_{0}^{1} R^{2} \mathrm{~d} z$

provides, equally well, an estimate of the increase in tensile modulus due to oriented rigid-fiber reinforcement (cf. Russel and Acrivos [13]). Thus, if the stress ratio $h$ in eqn. (37) is an inherently small quantity for a polymer melt, one obtains a fluid that might exhibit (desirably) smaller particle-stress effects in certain processing operations than would be anticipated simply from the enhanced elastic modulus of the solid product. According to the present treatment, this effect is seen to derive quite simply from the shear-thinning behavior of the fluid in the regions of strong shearing adjacent to long slender particles and especially towards their ends, compounded by a possible tensile "stiffening" in the pure fluid. Thus, the tensile stress acting in the particles, which is effectively transmitted by shear from the fluid, can be relatively small compared to the tensile stress contribution of the fluid itself. It may be of some general interest to observe that this kind of mechanical effect bears some strong similarities to that which may occur in fiber-reinforced solids because of interfacial slipping or yielding in the solid matrix adjacent to a reinforcing fiber. It is also of interest to note in this connection that, for the power-law type of shear-thinning behavior, eqn. (38) predicts a weaker dependence of particle stress on axial ratio $\alpha$, for given $\phi$, than the Newtonian formula (40). (In fact, the axial-ratio dependence in eqn. (39) could quite naturally be absorbed into the stress-ratio factor of eqn. (37), as $\tau\{\alpha \dot{\epsilon}\}$.)

At present, there appears to be no complete experimental evidence for such an effect in fluids, although it is worth noting that Charrier and Rieger [29] have reported some experimental observations of the inlet pressure drop associated with the converging flow into circular dies, for a molten polymer containing chopped glass fibers (in the concentration range of zero to twenty percent by volume). To the extent that such a converging flow may represent a uniaxial extension ( $c f$. Metzner and Metzner [27], Kizior and Seyer, [4]) one might be led, on the basis of Newtonian fluid behavior, to expect enormous tensile-stress levels and, hence, inlet pressure drops caused by the presence of oriented fibers.

While the experiments of Charrier and Rieger do indeed indicate a perceptible effect of the suspended fibers on inlet pressure drop, it does not appear to be very pronounced or systematic, except at the highest flow rates studied; and,

\footnotetext{
* That several of the tensile measurements cited may not correspond exactly to steady ex tension does not drastically alter the above reasoning, as long as the extension-rate is slowly varying in time or, otherwise, expressible as a unique function of instantaneous extension rate, as in extensions with fixed histories [3].
} 
then, it only represents an increase in the stress levels by a factor of approximately two, as compared to the factors of ten or more that have been obtained for Newtonian fluids at much smaller fiber concentrations $[2,3]$. Owing, however, to the well known difficulties of assigning definite kinematics to such converging flows $[4,27]$, this type of experiment may at best provide only a qualitative test of theory, albeit a practically interesting application. *

Furthermore, we see by eqns. (20), (35) and (36), that, for the power-law fluid, the axial component of the inner velocity field is given by:

$u(r, z)=z\left[1-(R / r)^{\frac{-n}{n+1}}\right] /\left[1-(a R / b)^{\frac{-n}{n+1}}\right]$

which is formally $O(1)$ for $\alpha \rightarrow \infty$. The form of eqn. (41) suggests a greatly diminished influence of the particle on the velocity field of surrounding fluid, which leads one to expect that the higher-order corrections for eqn. (37) would be of a smaller order in $\alpha$ than for the Newtonian case.

Apart from the obvious experimental amenability of the above predictions, they have further theoretical implications. For, if the ratio in eqn. (31) is indeed small in magnitude, i.e. of the order of unity, then the assumptions on which the approximation in eqn. (30) is based are no longer valid in a quantitative way.

To make a quantitative prediction would require more knowledge about the stress distribution in the fluid, in effect, to evaluate the term $\langle\sigma\rangle_{\mathrm{f}}$ in eqn. (26). This, in turn, would necessitate some further developments of the basic theory, including a consideration of far-field effects in non-linear viscoelastic media, a subject which will be taken up in a later publication.

For the present, we merely note in closing here that eqn. (41) remains formally valid in the dilute limit, $b / a \rightarrow \infty$, corresponding to the isolated particle and representing a far-field velocity perturbation of the form $(R / r)^{-n /(n+1)}$, which is much weaker than the corresponding $\log (r / R)$ term for the Newtonian case. This leads one again to anticipate a greatly diminished particle influence and, hence, smaller perturbations on the basic far-field flow of eqn. (1). However, in the absence of a proper far-field theory and a more realistic rheological model than that of eqn. (35) for the far-field fluid behavior, this must be considered speculative.

\section{Acknowledgement}

This work, presented in part at the 1974 Society of Rheology, Winter Meeting, Indian Wells, California, was partially supported by Grant GK 38303 from the National Science Foundation.

\section{References}

1 G.K. Batchelor, The stress generated in a non-dilute suspension of elongated particles by pure straining motion, J. Fluid Mech., 46 (1971) $813-829$.

* In addition, it is not certain that a slender-body theory is strictly applicable to the experiments of Charrier and Rieger, since the axial ratios of their nominally "short" fibers are not specified. 
2 J. Mewis and A.B. Metzner, The rheological properties of suspensions of fibres in Newtonian fluids subjected to extensional deformation, J. Fluid Mech., 62 (1974) 593-600.

3 C.B. Weinberger and J.D. Goddard, Extensional flow behavior of polymer solutions and particle suspensions in a spinning motion, Int. J. Multiphase Flow, 1 (1974) 465.

4 T.E. Kizior and F.A. Seyer, Axial stress in elongation flow of fiber suspensions, Trans. Soc. Rheol., 18 (1974) 271-285.

5 R.G. Cox, The motion of long slender bodies in a viscous fluid, Part 1: General theory, J. Fluid Mech., 44 (1970) 791.

6 R.G. Cox, The motion of long slender bodies in a viscous fluid, Part 2 : Shear flow, J. Fluid Mech., 45 (1971) 625.

7 G.K. Batchelor, The stress system in suspensions of force-free particles, J. Fluid Mech., 41 (1970) 545.

8 N.S. Clarke, The force distribution on a slender twisted particle in a Stokes flow, J. Fluid Mech., 52 (1972) 781.

9 L.G. Leal, The motion of slender rod-like particles in a second-order fluid, J. Fluid Mech., 69 (1975) 305.

10 F.J. Lockett, Flow of polymer fluids containing chopped fibers, Part 1 : Internal mechanics, NPL Rept. Mater. Appl. 25, December 1972.

11 G.K. Batchelor, Slender-body theory for particles of arbitrary cross-section in Stokes flow, J. Fluid Mech., 44 (1970) 419.

12 J.P.K. Tillet, Axial and transverse flow past slender axisymmetric bodies, J. Fluid Mech., 44 (1970) $401-417$.

13 W.B. Russel and A. Acrivos, On the effective moduli of composite materials: Slender rigid inclusions at dilute concentrations, Z. Angew. Math. Phys., 23 (1972) 434-464.

14 B.D. Coleman and W. Noll, Foundations of linear viscoelasticity, Rev. Mod. Phys., 33 (1961) 239 .

15 C. Truesdell, The Elements of Continuum Mechanics, Springer-Verlag, New York, 1966.

16 H.F. Weinberger, Variational properties of steady fall in Stokes flow, J. Fluid Mech., 52 (1972) $321-344$.

17 A.C. Pipkin, Lectures on Viscoelasticity Theory, Springer-Verlag, New York, 1972.

$18 \mathrm{R}$. Tanner and $\mathrm{G}$. Williams, Iterative numerical methods for some integral equations arising in rheology, Trans. Soc. Rheol., 1 (1970) 19.

20 B.D. Coleman, H. Markowitz and W. Noll, Viscumetric flows of Non-Newtonian Fluids, Springer-Verlag, New York, 1966.

21 J.L. Ericksen, Overdetermination of speed in rectilinear motion of non-Newtonian fluids, Q. Appl. Math., 14 (1956) 318.

22 D.E. Stone, On the non-existence of rectilinear motion in plastic solids and non-Newtonian fluids, Q. Appl. Math., 15 (1957) 257.

23 A.G. Fredrickson, Principles and Applications of Rheology, Prentice-Hall, Englewood Cliffs, N.J., 1964.

24 A.E. Green and R.S. Rivlin, Steady flow of non-Newtonian fluids through tubes, Q. Appl. Math., 14 (1956) 299.

25 A.S. Wineman and A.C. Pipkin, Viscoelastic flow in titled troughs, Acta Mech., 2 (1966) 104.

$26 \mathrm{~J} . \mathrm{D}$. Goddard and C.Miller, Non-linear effects in the rheology of dilute suspensions, $\mathrm{J}$. Fluid Mech., 28 (1967) 657.

27 A.B. Metzner and A.P. Metzner, Stress levels in rapid extensional flows of polymer fluids, Rheol. Acta, 9 (1970) 174-181.

28 I.J. Chen, G.E. Hagler, L.E. Abbott, D.C. Bogue and J.L. White, Interpretation of tensile and melt spinning experiments on low density and high density polyethylene, Trans, Soc. Rheol., 16 (1972) 473.

29 J.-M. Charrier and J.M. Rieger, Flow of short glass fibre-filled polymer melts, Fibre Sei. and Technol., 7 (1974) 161-172. 